\title{
Promotion or Inhibition of IAPP Aggregation by Zinc Coordination Depends on Its Relative Concentration
}

\author{
Praveen Nedumpully-Govindan ${ }^{\mathrm{a}}$, Ye Yang ${ }^{\mathrm{b}}$, Rachel Andorfer ${ }^{\mathrm{a}}$, Weiguo Cao ${ }^{\mathrm{b}}$, and Feng Ding ${ }^{\mathrm{a}, 1}$ \\ ${ }^{a}$ Department of Physics and Astronomy, Clemson University, Clemson, SC 29634 \\ ${ }^{\mathrm{b}}$ Department of Biochemistry and Genetics, Clemson University, Clemson, SC 29634 \\ ${ }^{1}$ Corresponding Author: fding@clemson.edu; Tel: 864-656-0459
}

\section{Supplementary Tables}

Table S1. Zinc-Imidazole bond parameters. The interaction parameters were obtained from statistical analysis of zinc-histidine binding in PDB (Fig. S1). An infinite square-well potential bound by dmin and dmax was used constrain bond lengths (Fig. S2). The values of $d_{\min }$ and $d_{\max }$ were obtained by fitting the highest peak in the histograms of Fig. S2. In the case of multiple hIAPPs binding to a zinc ion, His-zinc-His angle was also maintained by applying additional constraints between NE2 atoms of His residues. The NE2-NE2 ideal lengths were calculated using the NE2-zinc coordination bond lengths and ideal tetrahedral $\left(109.5^{\circ}\right.$, for dimer, trimer and tetramer hIAPP coordinated by zinc) or octahedral angles $\left(90^{\circ}\right.$ and $180^{\circ}$, for hexamer hIAPP coordinated by zinc).

\begin{tabular}{|l|l|l|}
\hline Bond & $\mathbf{d}_{\min }(\mathbf{\AA})$ & $\mathbf{d}_{\max }(\AA)$ \\
\hline Zn-NE2 & 2.04 & 2.20 \\
\hline Zn-ND1 & 4.08 & 4.30 \\
\hline Zn-CD2 & 2.98 & 3.16 \\
\hline Zn-CE1 & 2.96 & 3.16 \\
\hline Zn-CG & 4.12 & 4.28 \\
\hline NE2-NE2 (tetrahedral, $\left.\mathbf{1 0 9 . 5}^{\circ}\right)$ & 3.33 & 3.59 \\
\hline NE2-NE2 (octahedral, $\left.\mathbf{9 0}^{\circ}\right)$ & 2.89 & 3.11 \\
\hline NE2-NE2 (octahedral, $\left.\mathbf{1 8 0}^{\circ}\right)$ & 4.08 & 4.40 \\
\hline
\end{tabular}




\section{Supplementary Figures}

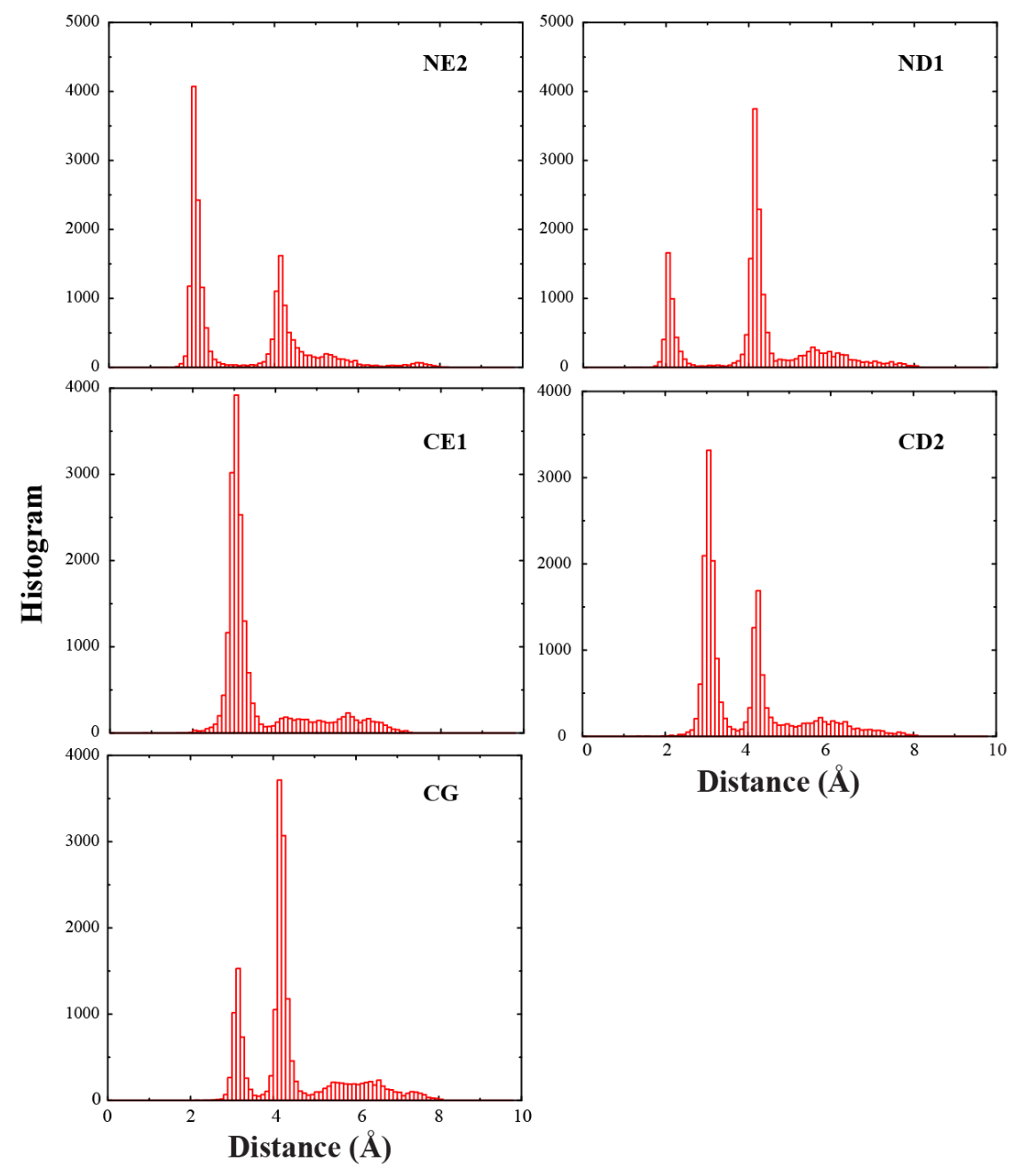

Figure S1. Zn-His coordination bond lengths. The bond length distribution for the coordination bond between zinc and histidine imidazole ring atoms. The distribution is obtained using 9547 zinc containing structures in the PDB. Zinc can bind to either NE2 or ND1 atom of imidazole, giving raise to two peaks. The zinc-NE2 binding seems to be more common as seen in the peak values and hence only zinc-NE2 binding, with a coordination bond length of $\sim 2.1 \AA$, was considered in simulations. Because of the rigid nature of coordination bond, the distance with the other imidazole atoms also has specific values. We chose the distance corresponding to the highest peak in our model (Table S1). 

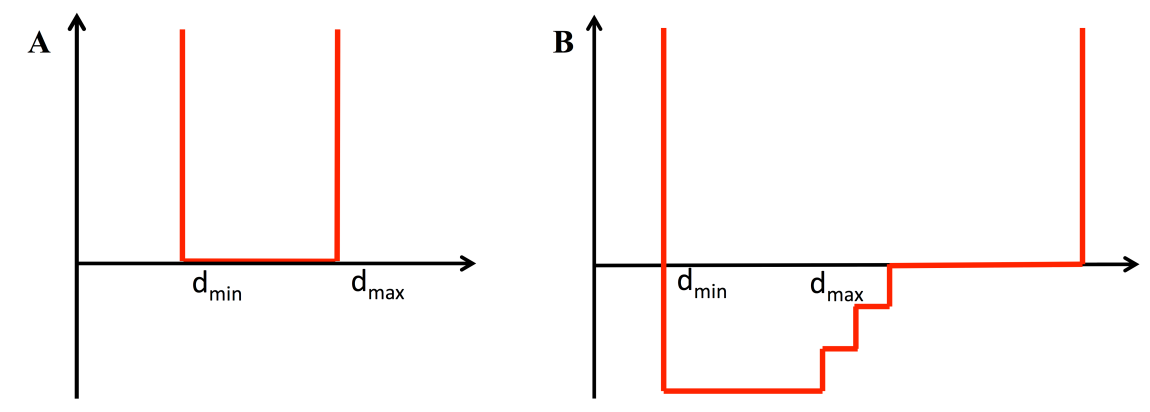

Figure S2. Modeling Zn-His coordination bond. (A) Schematic of the square-well potential used to model coordination bonds between zinc ion and imidazole atoms. dmin and dmax values obtained from Fig. S1 are listed in Table 1. (B) At the beginning of each simulation, a 10,000 steps long simulation is performed with a weak attractive potential between zinc and NE2 atoms of His 18. This step is necessary to bring the histidines close to zinc. 


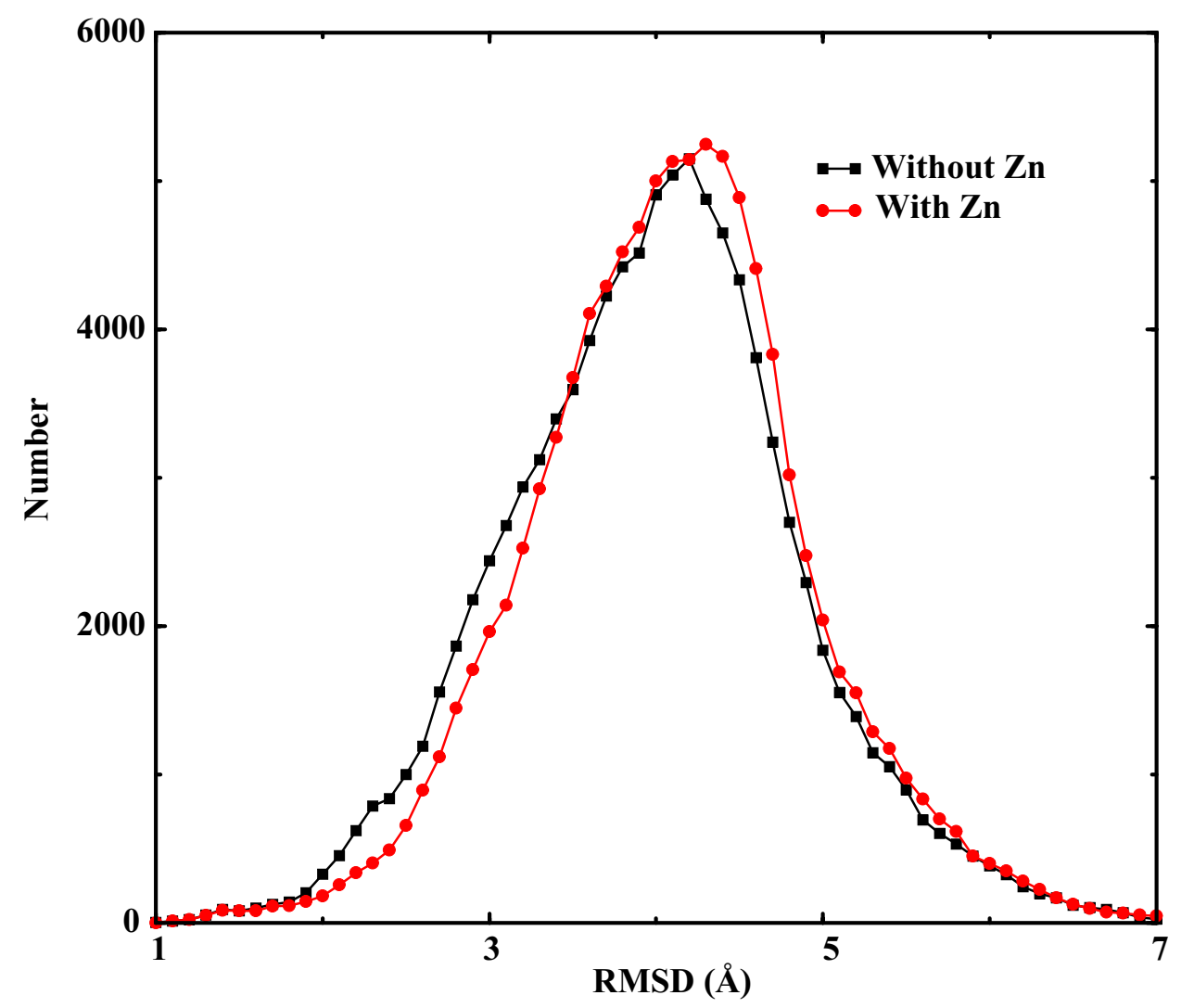

Figure S3. The effect of zinc binding on hIAPP1-19. The distribution of RMSD values of hIAPP1-19 with and without zinc binding. When zinc ion binds the His18 residues, a small shift of RMSD curve to the right is observed, likely due to electrostatic repulsion between zinc ions and two positively charged residues (Lys1 and Arg11). 


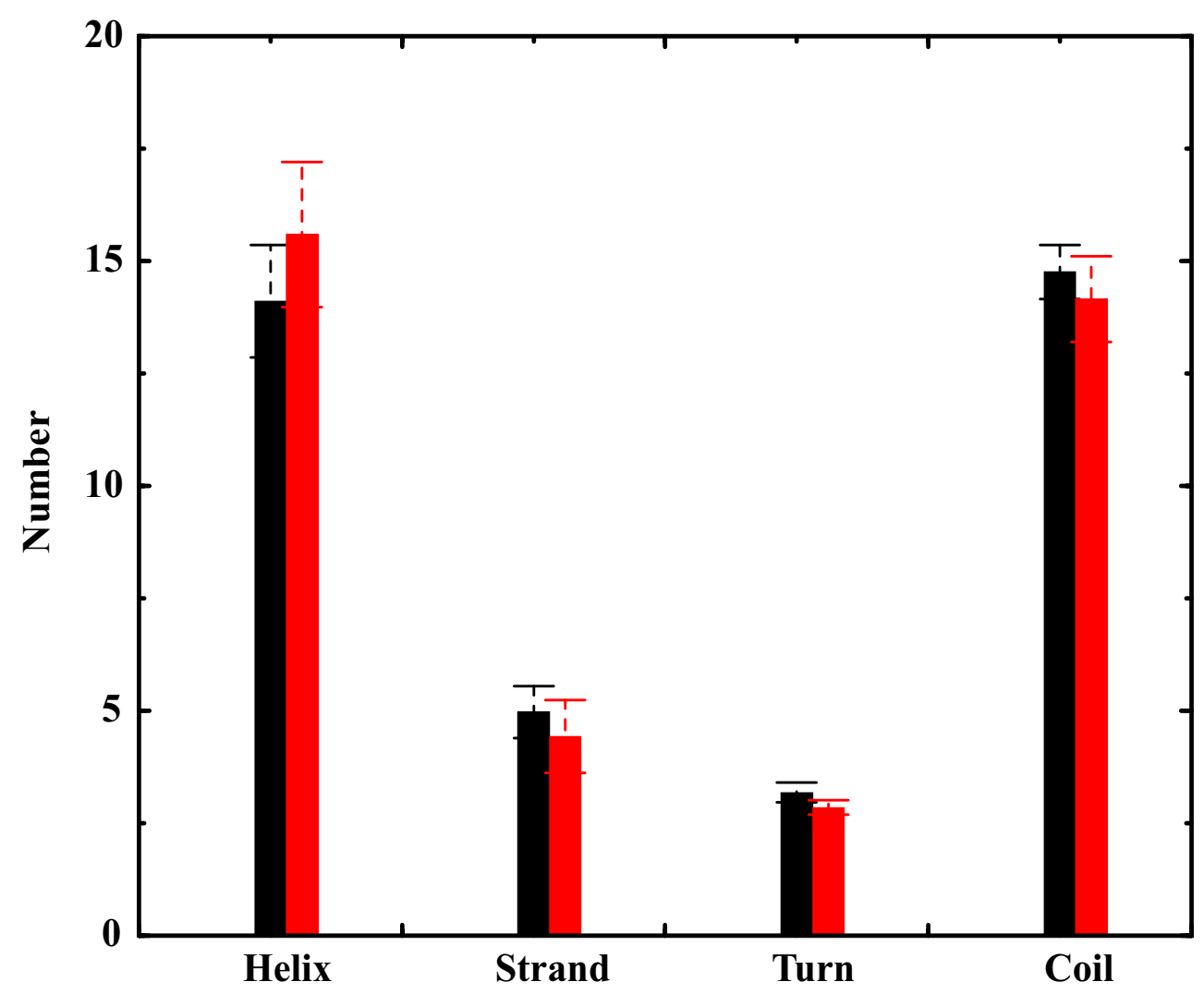

Figure S4. The effect of zinc binding on hIAPP1-37. The secondary structure contents of fulllength hIAPP monomers with (red) and without (black) a bound zinc ion. In the zinc-bound case, a small increase of helical content and a decrease of coil content are observed. The error bars represent standard error of measurements from ten independent simulations. 


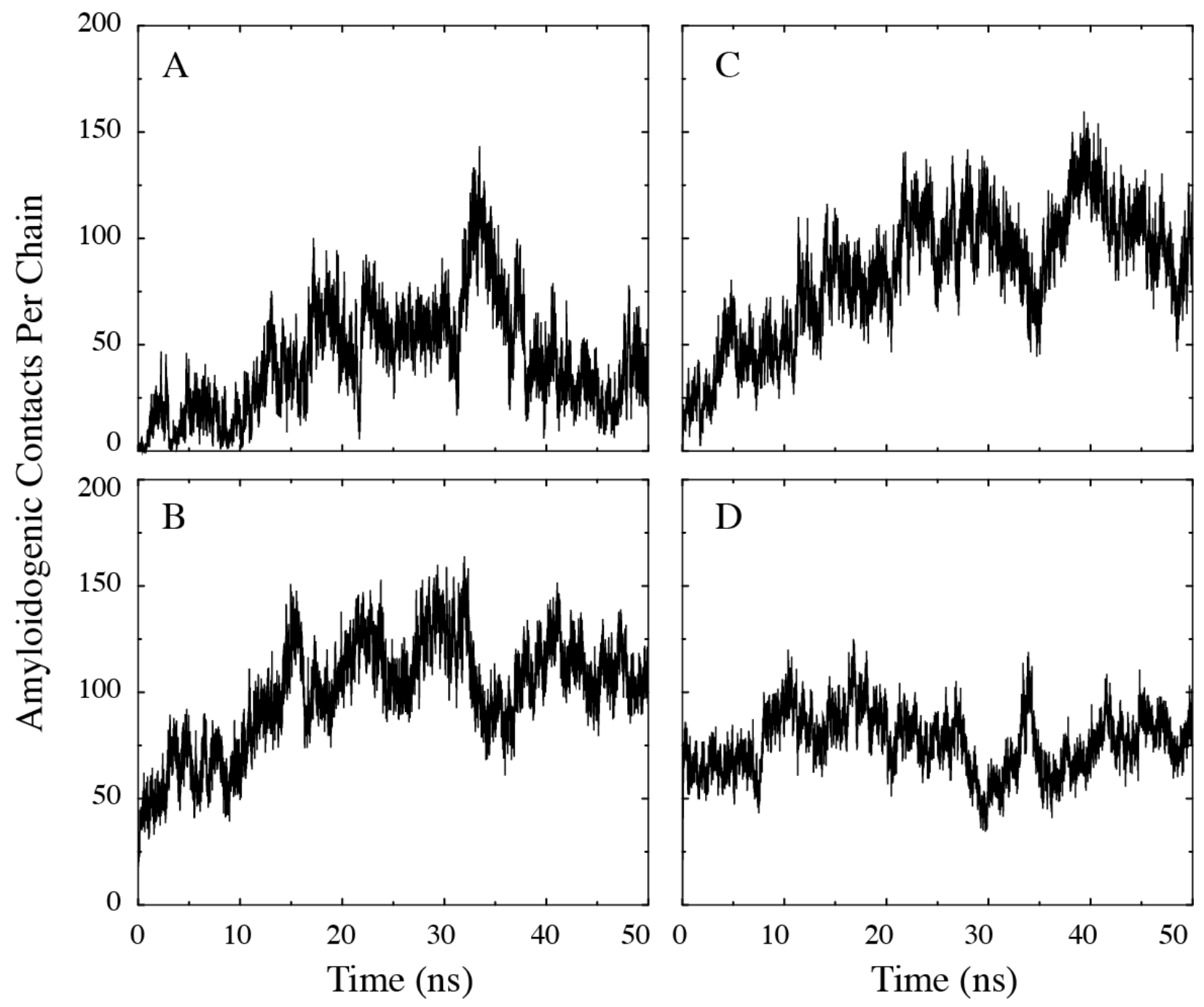

Figure S5. Time dependent amyloid contact number. The amyloid contact number per chain for different systems as a function of time for simulations of (A) hI2-Zn, (B) hI3-Zn, (C) hI4-Zn, and (D) hI6-Zn. The instantaneous contact number is averaged over 10 independent simulations. 

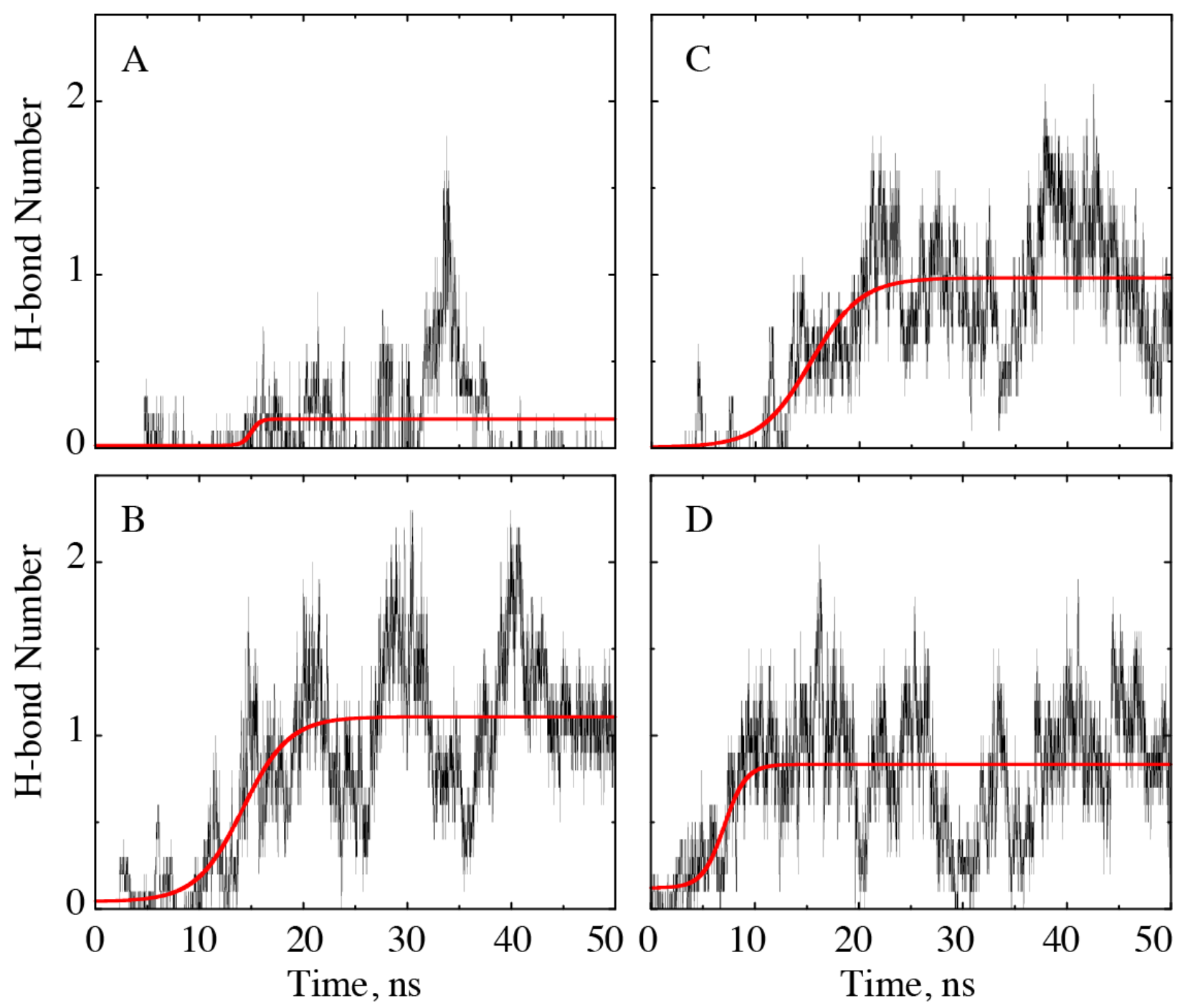

Figure S6. Time dependence of hydrogen bond number on time. The inter-chain backbone hydrogen bond number is plotted as a function of time for simulations of (A) hI2-Zn, (B) hI3-Zn, (C) hI4-Zn, and (D) hI6-Zn. The hydrogen bond number is averaged over 10 independent DMD simulations. The red lines correspond to the sigmoidal fitting using Eq. 1. The corresponding fitted values are listed in Table 1. 

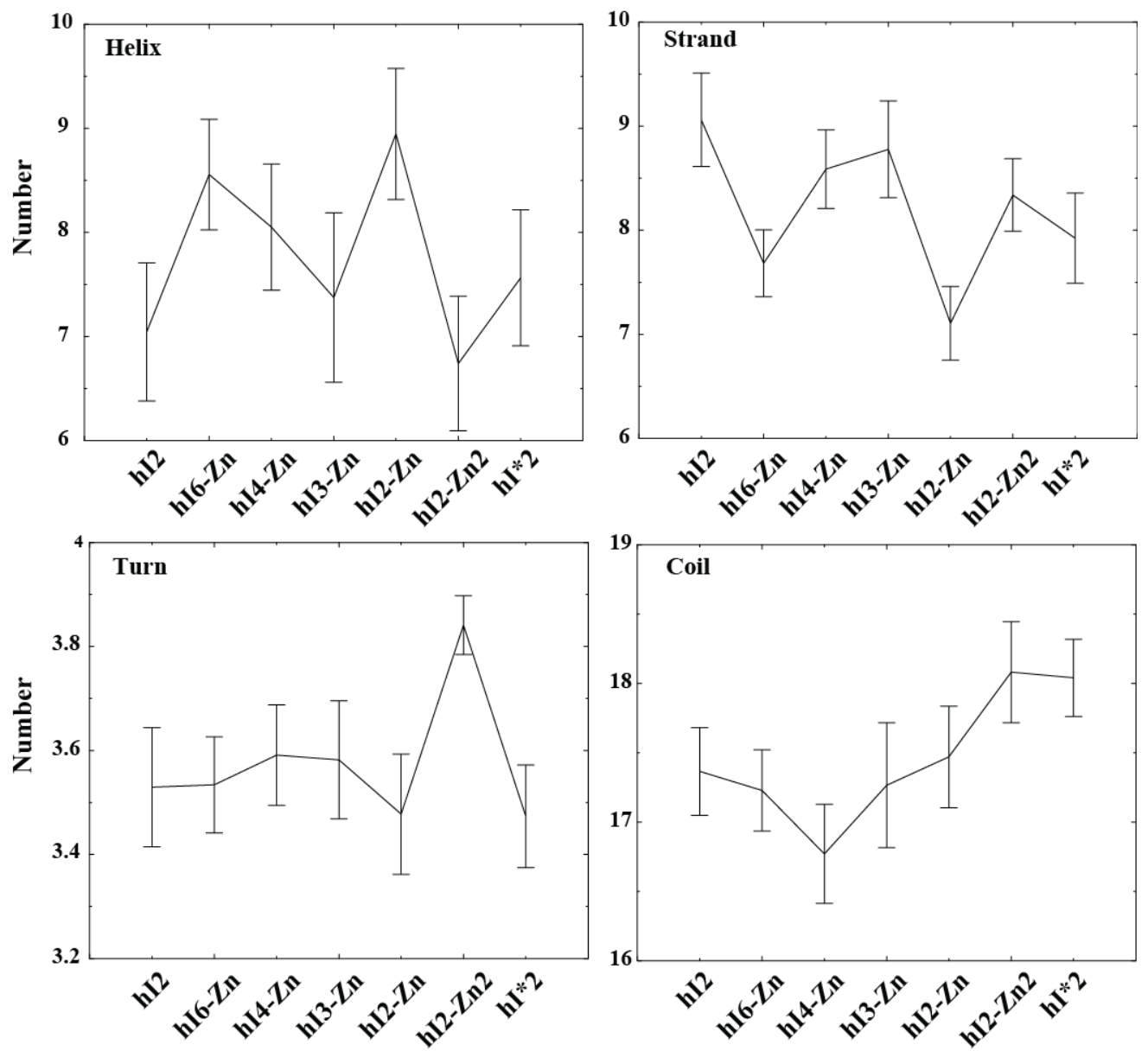

Figure S7. Secondary structure contents of different zinc-coordinated hIAPP oligomers. The total secondary structure contents (per chain) for different oligomers coordinated by zinc ion. Unlike the contact number, or local hydrogen bonds, secondary structure contents show no clear trend as a function of coordination number. 